Gastroenterologe 2020 · 15:527-529

https://doi.org/10.1007/s11377-020-00491-2

() Springer Medizin Verlag GmbH, ein Teil von Springer Nature 2020

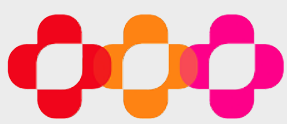

netzwerk

gegen darmkrebs
Netzwerk gegen Darmkrebs e.V.

Arabellastr. 27, 81925 München

Tel. 089/9250 1748

Geschäftsstelle:

Kathrin Schmid-Bodynek

kathrin.schmid-bodynek@netzwerk-gegen-darmkrebs.de

www.netzwerk-gegen-darmkrebs.de

Redaktion: V.i.S.d.P.

Dr. Georg Ralle, München

\title{
Risikoadaptierte Darmkrebsvorsorge
}

\section{Bayerisches Versorgungsprojekt FARKOR auf der Zielgeraden}

Das FARKOR-Projekt soll für Menschen mit familiär erhöhtem Darmkrebsrisiko einen gesetzlichen Anspruch auf eine zeitlich vorgezogene Früherkennung erwirken. Dr. Berndt Birkner, Mitinitiator des Projekts, appelliert an niedergelassene Ärzte, sich auch jetzt noch einzuschreiben.

„Menschen mit familiär erhöhtem Darmkrebsrisiko erkranken häufiger und deutlich früher an Darmkrebs als der Bevölkerungsdurchschnitt“, sagt Dr. Berndt Birkner, Gastroenterologe in München. „Die aktuellen Altersgrenzen für das Screening sind für diese Personen zu spät angesetzt, und wir brauchen eine andere Strategie, um sie zu schützen."

Im bayerischen Modellprojekt FARKOR wird daher bei Personen im Alter von 25 bis 49 Jahren evaluiert, ob sich anhand einer strukturierten Familienanamnese ein erhöhtes Risiko sicher identifizieren lässt. „Wenn dem so ist, werden wir bei der Koloskopie dieser Risikopersonen gehäuft Läsionen finden“, erläutert Birkner. Die Maßnahme sei daher essenziell, um die strukturierte Anamnese zu validieren.

Zuletzt ist das Projekt durch SARS-CoV-2 jedoch etwas ins
Stocken geraten, Birkner zufolge sind die Zuweisungen an koloskopierende Ärzte seit Beginn der Pandemie deutlich zurückgegangen. „Wenn es so weitergeht, dann erreichen wir die bis Ende März 2021 vorgesehenen 1800 Koloskopien nicht, und die statistische Aussagekraft der Studie wäre eingeschränkt."

Birkner appelliert daher an niedergelassene Kolleginnen und Kollegen, sich auch jetzt noch in das Projekt einzuschreiben. Teilnehmen können Ärzte aller Fachrichtungen, Voraussetzung ist ein Sitz in Bayern sowie eine Fortbildung zur Familienanamnese. „Sie kann online absolviert werden und gibt Fortbildungspunkte“, sagt Birkner. „Darüber hinaus werden sämtliche Leistungen attraktiv vergütet." Birkner hofft, dass auf der Zielgeraden noch einige 100 Koloskopien hinzukommen und die Evaluation erfolgreich ist. „Denn dann hat die Maßnahme die Chance, in die Regelversorgung überzugehen, sodass jüngere Erwachsene mit erhöhtem Risiko nicht mehr das Nachsehen haben."

https://www.kvb.de/abrechnung/verguetungsvertraege/farkor/

Autor: Günter Löffelmann

\section{Organisiertes Darmkrebsscreening}

\author{
Vom Start weg holprig
}

Die Ausgestaltung des Darmkrebsscreenings hat massive konzeptionelle Schwächen. Die für den Erfolg notwendige Teilnahmerate von mindestens $60 \%$ lässt sich damit nicht erreichen.

Seit April 2019 gibt es in Deutschland das organisierte Darmkrebsscreening. Oder sollte man besser das ,desorganisierte sagen? Der Begriff liegt nahe, wenn man sich die Ausgestaltung des Programms näher ansieht.

Die Probleme begannen bereits beim Versand der Einladungen. Anders als bei der Brustkrebsfrüherkennung erfolgt er dezentral, mit der Folge, dass die ersten Einladungen nicht wie vorgesehen ab 1. Juli an die Versicherten gingen, sondern erst $\mathrm{ab}$ dem Herbst 2019. Ein geringer Zeitverlust, angesichts der Verspätung, mit der das Programm vom G-BA auf den Weg gebracht wurde. Viel schwerer wiegen andere Schwachstellen, allen voran das Einladungsverfahren.

In seiner jetzigen Form wirft es Versicherten eher Knüppel zwischen die Beine, als dass es sie zur Teilnahme motiviert. Wer hat schon Lust, sich den Stuhltest vom Arzt abzuholen, ihn zu Hause durchzuführen, ihn wieder zum Arzt zurückzubringen, und die Praxis dann noch mal zu kontaktieren, um das Ergebnis zu erfahren. Dieses Procedere erfordert schon ein gehobenes Maß an
Motivation und Initiative selbst bei jenen Versicherten, die der Krebsvorsorge prinzipiell aufgeschlossen gegenüberstehen.

Ein weiterer Planungsfehler besteht darin, dass jeder Stuhltest zugelassen ist, der die Mindestanforderungen an Sensitivität und Spezifität in einer einzigen Studie erfüllt hat. Die Folge ist, dass derzeit etwa 15 verschiedene Tests auf dem Markt sind, deren Positivitätsrate zwischen fünf und $25 \%$ schwankt. Tests mit hoher Positivitätsrate produzieren vermutlich eine relativ hohe Zahl an falsch positiven Ergebnissen, sodass sich die getesteten Personen unter Umständen unnötig einer Koloskopie unterziehen.

Es ist müßig darauf hinzuweisen, dass sich der GBA bei der Ausgestaltung des organisierten Darmkrebsscreenings am Modell in den Niederlanden hätte orientieren können. Dieses ist wissenschaftlich gut fundiert, zentral organisiert, es gibt einen einzigen Test, den die Teilnehmer mit der Post nach Hause geschickt bekommen, sie können ihn ebenfalls per Post ans Labor schicken und erhalten von dort das Ergebnis. So einfach kann es gehen.

Zur Erinnerung: Damit ein Krebsfrüherkennungsprogramm effizient ist, müssen mindestens $60 \%$ der Anspruchsberechtigten teilnehmen. In den Niederlanden werden seit Jahren Raten über $70 \%$ erreicht, in Deutschland 
zwischen zehn und $15 \%$. Und es ist nicht davon auszugehen, dass unser derzeitiges Programm daran viel ändern wird.

Der Netzwerk gegen Darmkrebs e. V. wird daher weiter auf Änderungen drängen, um auch in Deutschland noch zu einer guten Lösung für das Darmkrebsscreening zu kommen. Darüber hinaus setzt sich der Verein dafür ein, über den Einsatz innovativer Technologien nachzudenken und sie im Rahmen von Pilotprojekten zu testen. Als Beispiel sei an dieser Stelle ein Stuhltest genannt, den Probanden in Kombination mit einer App selbst aus- werten können. Ärzte und Krankenkassen sollten in einen intensiven Austausch treten, um solche innovativen Verfahren zu etablieren. Sie erleichtern den Menschen damit die Krebsvorsorge und können die Dienstleister im Gesundheitssystem erheblich entlasten - nicht nur in Zeiten der Corona-Pandemie ein wichtiges Kriterium.

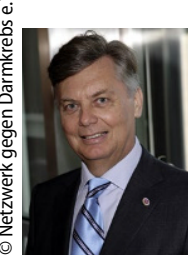

Autor: Dr. Berndt Birkner, Vizepräsident des Netzwerk gegen Darmkrebs e.V.

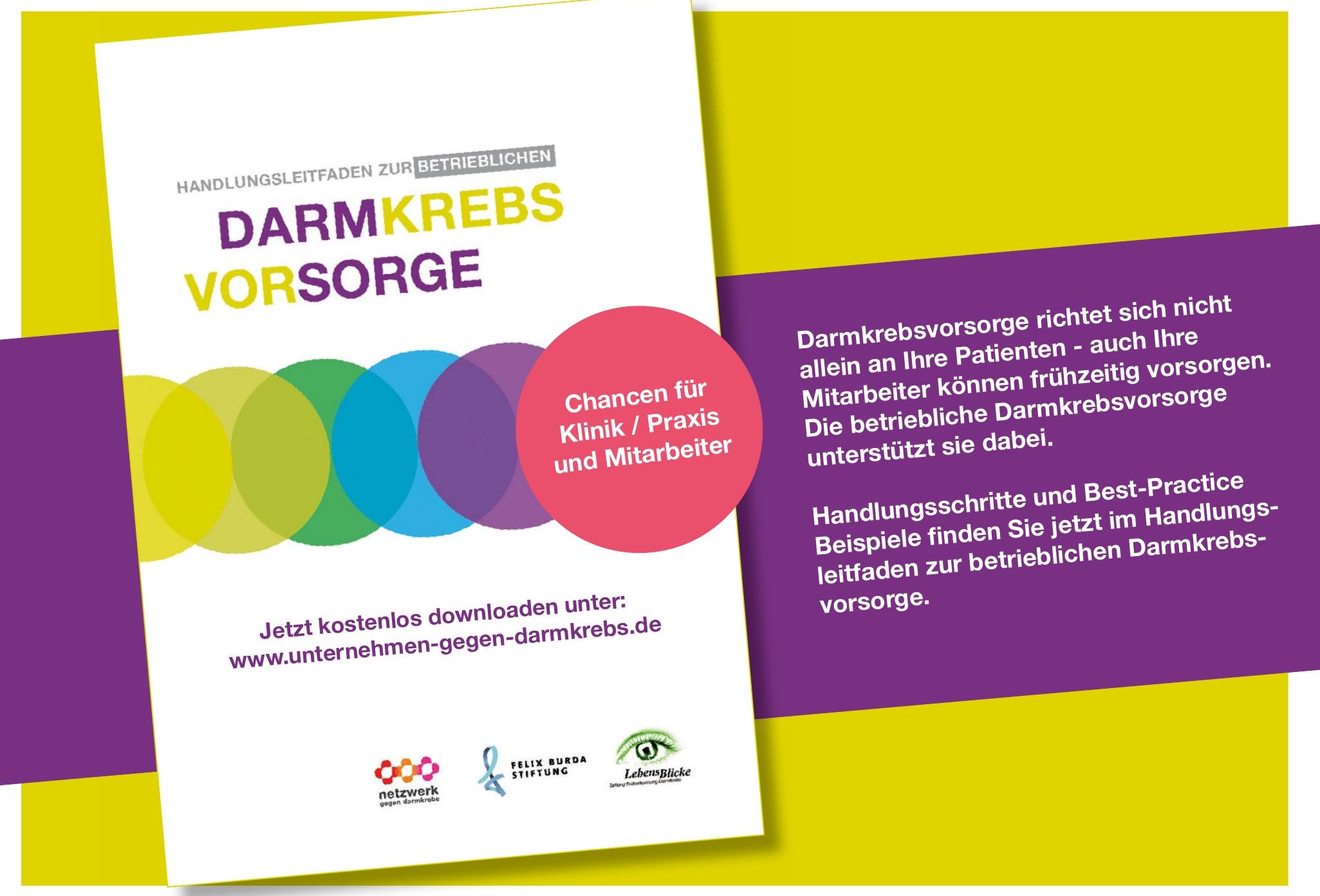




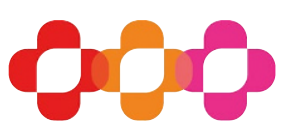

netzwerk

gegen darmkrebs

Das Netzwerk gegen Darmkrebs

unterstützt FARKOR

als Kooperationspartner.

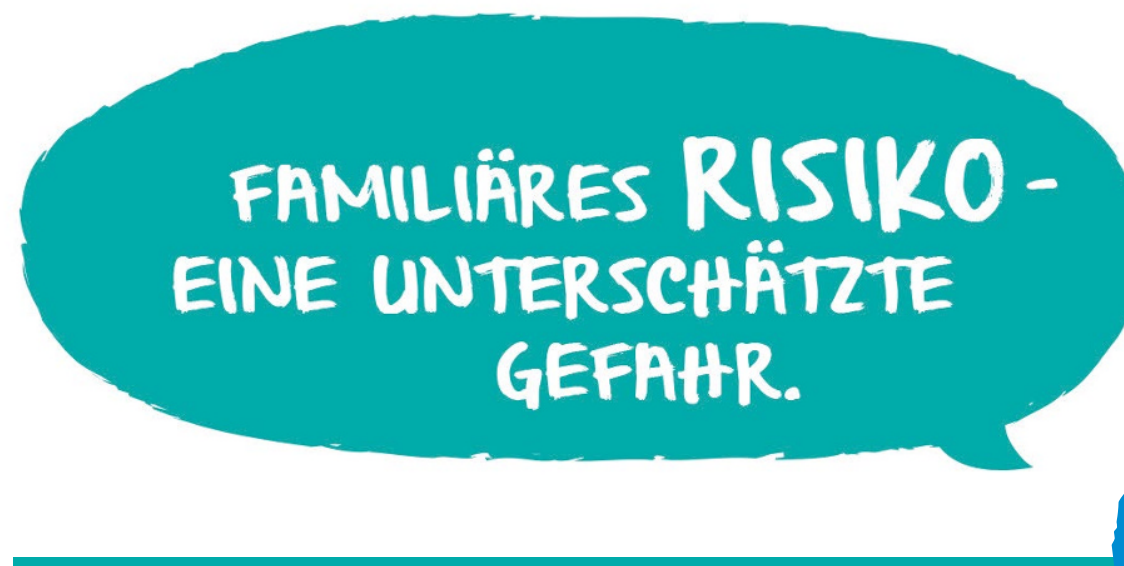

\section{sprich}

drüber!
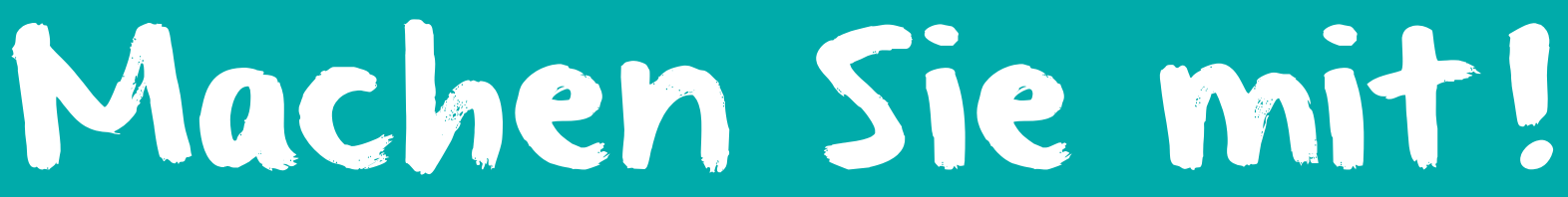

FARKOR ist ein bayerisches Modellprojekt, das familiären Darmkrebs verhindern möchte.

Deshalb an alle Gastroenterologen in Bayern:

Machen Sie mit!

Es lohnt sich für Sie und Ihre Patienten. 